\title{
NAVIER-STOKES-BRINKMAN SYSTEM FOR INTERACTION OF VISCOUS WAVES WITH A SUBMERGED POROUS STRUCTURE
}

\author{
LEMI GUTA AND S. SUNDAR
}

\begin{abstract}
In this paper the interaction of a two-dimensional progressive wave train over a submerged rectangular porous breakwater is studied theoretically. For this purpose, the time dependent incompressible Navier-Stokes-Brinkman system is newly derived for wave propagating over submerged breakwater. A staggered grid Finite Volume Method (FVM) is used to solve the Navier-Stokes-Brinkman system. The free surface boundary condition and the interfacial boundary conditions between the water and porous media are in complete form. A Volume-of-Fluid (VOF) methodology is employed to track the free surface. The validity of present model is verified based on the compressions with the existing experimental results. Having verified the accuracy of the numerical model, the effects of several parameters of a wave and a submerged breakwater were systematically investigated. The results indicate that the wave transformations are affected primarily by the breakwater width and the structure permeability. The numerical results also illustrate that for the porous breakwater, an optimum porosity value seems to exist at which the transmitted wave height is minimized. It has been observed that the transmission coefficient decreases as the permeability increases. However, the results indicate that further increase of the structure permeability beyond the optimal value increase the transmission coefficient.
\end{abstract}

\section{Introduction}

Study of wave-porous structure interaction is becoming increasingly important for problems like design of porous breakwaters to protect harbors, pillows holding open sea platforms, erosion of the seacoast etc. The Numerical Prediction of Performance of submerged breakwater was studied by A.Chiranjeevi [2] The accurate prediction and estimation of the reflection, transmission and deformation of water waves and their interaction with structures are important for design purposes of porous breakwaters like rubble-mound breakwaters in ports and coastal areas. In recent years there have been

Corresponding author: Lemi Guta.

Received June 2, 2009.

Key words and phrases. Finite volume method, viscous waves, free surface flow, submerged porous breakwater, free surface flow. 
many techniques developed for the numerical solution of complicated problems in fluid dynamics. The simulation of the nonlinear dynamic interactions between waves, a submerged breakwater and the seabed was studied by Dong-Soo Hur [7]. The interaction of a solitary wave and a submerged dike was studied by Ching-Jer Huang and Chih-Ming Dong [4]. The use of porous plate structures as wave makers has been studied by Chwang [3]. With the development activities, the dynamic equilibrium of the coastal region is disturbed resulting in coastal erosion and accretion. Coastal erosion is a severe problem worldwide, threatening the coastal properties, degradation of valuable land and natural resources, disruption to fishing, shipping and tourism. The development of coastal facilities has necessitated proper management of the sea front warranting for construction of coastal protective structures. The choice of the structure would depend on the wave environment and the morphology of the coastal region. Submerged structure has been employed to protect coastal environments for many years. As water wave propagate over submerged structures on the bottom, waves are partly reflected and partly diffracted, as the result the water elevation decreases behind submerged structures. Our work is to establish a numerical model to investigate the interaction of a two-dimensional progressive wave train over a submerged porous breakwater incorporating free surface. This paper is organized as follows. In section 2, we present a mathematical model, describing the interaction of nonlinear progressive viscous waves with porous breakwater and boundary conditions. The Navier-Stokes-Brinkman system is employed to simulate the transport quantities and to solve the free surface flow problem. In section 3, a numerical scheme is described. A Volume-of-Fluid (VOF) methodology is employed to track the free surface. In section 4, some numerical tests are presented. Numerical results for the wave interaction with a submerged breakwater are discussed and compared with the existing experimental results. The effect of structure permeability, width and height on the water elevation while interacting with the different types of porous obstacle at high Reynolds number are considered.

\section{Governing the problem}

We have considered the interaction of a gravity wave train of height $H$, with porous submerged rectangular breakwater surrounded by the fluid of finite depth $d$, the width and height of the structure are denoted by $w$ and $h$ respectively as shown in Figure 1. The problem is restricted to two dimensions. Furthermore, momentum conservation can be described by incompressible isothermal Navier-Stokes equations for the flow in pure liquid zone, and Brinkman (extension to Darcy) model for flow in porous zone.

\subsection{Flow in the fluid region}

The liquid we are modelling is incompressible and the governing equation in the fluid region is given by incompressible Navier-Stokes Equation

$$
\frac{\partial \mathbf{u}}{\partial t}+(\mathbf{u} \cdot \nabla) \mathbf{u}=\frac{1}{\rho}\left(\mu \Delta \mathbf{u}-\nabla p+f_{N S}\right)
$$




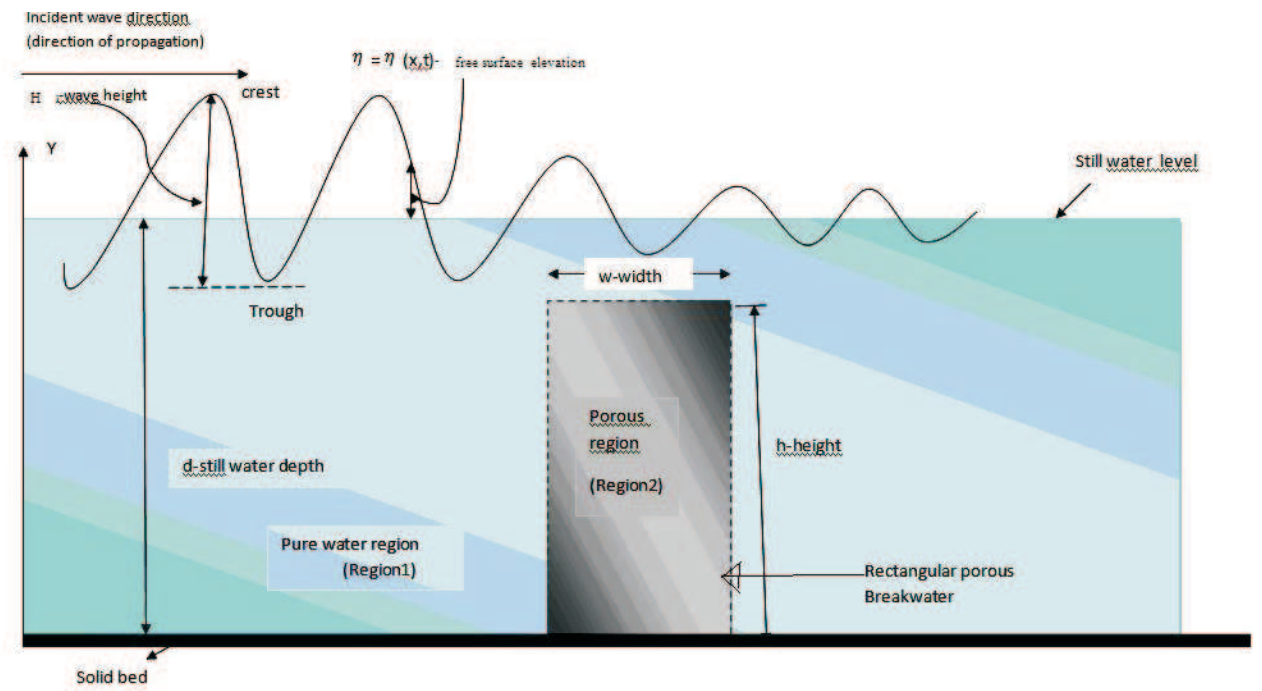

Figure 1: Schematic diagram of a non-linear wave passing over a rectangular porous structure

$$
\nabla \cdot \mathbf{u}=0
$$

where $\mathbf{u}=(u, v)^{T}$ is velocity vector, $p$ is the hydrodynamic pressure, $\rho$ is density, $f_{N S}$ is external body force and $\mu$ is the dynamic viscosity coefficient. Equations (1) and (2) can be equivalently expressed as $u$ and $v$ component of momentum equations in the following way

$$
\begin{aligned}
& \frac{\partial u}{\partial t}+\frac{\partial u^{2}}{\partial x}+\frac{\partial u v}{\partial y}+\frac{\partial \tilde{p}}{\partial x}=\nu\left(\frac{\partial^{2} u}{\partial x^{2}}+\frac{\partial^{2} u}{\partial y^{2}}\right)+f_{x} \\
& \frac{\partial v}{\partial t}+\frac{\partial u v}{\partial x}+\frac{\partial v^{2}}{\partial y}+\frac{\partial \tilde{p}}{\partial y}=\nu\left(\frac{\partial^{2} v}{\partial x^{2}}+\frac{\partial^{2} v}{\partial y^{2}}\right)+f_{y}
\end{aligned}
$$

The $x$ and $y$ coordinate axes are horizontal and vertical components of spatial coordinates respectively, with the origin located at the lower left of the computing region and the kinematics viscosity coefficient is given by $\nu=\frac{\mu}{\rho}$. The corresponding components of the velocities are $u$ and $v$ in the Cartesian coordinates $(x, y)$. $\mathrm{t}$ is the time, while $\tilde{p}$ is the ratio of the pressure to the constant density. (For brevity, we usually refer to as $\tilde{p}=p$ "pressure"). The $x$ and $y$ components of body force are denoted by $f_{x}$ and $f_{y}$ respectively.

\subsection{Flow in porous media}

By porous medium we mean a material consisting of a solid matrix with an interconnected void, the interconnectedness of the void (the pores) allows the flow of one or more 
fluids through the material. Examples of porous media are soil, bread, paper etc., the details of the internal geometry of porous media are essentially random and unknown [12]. The saturated flow in porous media is described by Darcy's model and expressed by the equations

$$
\begin{gathered}
\mu K^{-1} \mathbf{u}=f_{D}-\nabla p \\
\nabla \cdot \mathbf{u}=0
\end{gathered}
$$

The permeability tensor, a characteristic of the porous media geometry, is denoted by $K$. The above relation does not take into account the viscous resistance and convective acceleration terms. This, in effect, imposes serious limitations on the range of validity of Darcy's law, which was valid for flows with low Reynolds number. As the flow velocity or particle size increases, Darcy's linear relationship between the discharge velocity and pressure gradient breaks down. Accordingly, in order to account for the high porosity and high velocity, the following Brinkman model, which is an extension of Darcy's model has been considered. Brinkman accounted for the presence of a solid boundary by adding a viscous term to Darcy's law. The unsteady Brinkman equation for viscous flows in a porous media is given as

$$
\begin{gathered}
s \frac{\partial \mathbf{u}}{\partial t}+(\mathbf{u} \cdot \nabla) \mathbf{u}=\frac{1}{\rho}\left(\nabla \cdot\left(\mu_{e f f} \nabla \mathbf{u}\right)-\mu K^{-1} \mathbf{u}-\nabla p+f_{B}\right) \\
\nabla \cdot \mathbf{u}=0
\end{gathered}
$$

where $\mu_{\text {eff }}=\frac{\mu}{\epsilon_{p}}$ is effective viscosity,$s=1+\left(1-\epsilon_{p}\right) c_{m} / \epsilon_{p}$ is the inertial coefficient, $c_{m}$ is a coefficient of added mass and $\epsilon_{p}$ is the porosity of the porous structure. The left hand side terms in equation (7) denote the inertial force per unit volume, the writehand-side terms represent the total forces acting on the fluid per unit volume. The first term on the right- hand-side is the force caused by the pressure gradient. The second term denotes the viscous force and can be traced back to Brinkman who suggests that for flow motion in porous media with high permeability, the viscous shear stresses acting on the pore flow should be added to the momentum equation. The viscous stress term is responsible for transferring shear force and may become increasingly important near the interface between the porous and pure liquid regions. The convective term should improve the non-linear effect for the wave structure interaction, while the benefit of including the viscous force term for pore flow is the complete forces acting on the flow are taking into account. The third term represents the resistance force acting on flow by fixed solid skeleton. Consequently, the equations are of the same type as the Navier-Stokes equations for the flow in the pure liquid region. Hence, both equations can be solved using the same numerical algorithm and formulated as single system called Navier-Stokes-Brinkman system. 


\subsection{Dimensionless form of Navier-Stokes-Brinkman system}

Experimental studies of flows are often carried out on models, and the results are displayed in dimensionless form, thus allowing scaling to real flow conditions. The same approach can be undertaken in numerical studies as well. The governing equations

$$
\tilde{s} \frac{\partial u}{\partial t}+(u \cdot \nabla) u=\frac{1}{\rho}\left(\mu \nabla \cdot(\tilde{\mu} \nabla u)-\mu K^{-1} u-\nabla p+\tilde{f}\right)
$$

can be transferred to dimensionless form by using appropriate normalization. Let us define dimensionless coordinates $x_{*}$ and $t_{*}$ and variables $u_{*}$ and $p_{*}$ as: $t_{*}=\frac{t}{T}, x_{*}=\frac{x}{d}$ , $u_{*}=\frac{u}{U}, p_{*}=\frac{p}{\rho U^{2}}, \tilde{\mu_{*}}=\frac{\mu}{\tilde{\mu}}, f_{*}=\frac{f}{\rho g}$ where $U$ is the characteristics velocity and $d$ is a length scale. If the velocity and the length are non-dimensionalized by $U$ and $d$ where $U=\frac{c H_{i}}{d}$, c is the phase speed, and $H_{i}$ is the $i^{t h}$ wave height of the incident waves, and $T=\frac{d}{U}$ is chosen to non-dimensionalized the time. After changing $(u, p)$ to $\left(u_{*}, p_{*}\right)$ and multiplying the resulting momentum equations by $\frac{d}{\rho U^{2}}$.

$$
\begin{gathered}
\frac{\tilde{s} d}{U T} \frac{\partial u_{*}}{\partial t_{*}}-\frac{\mu}{\rho d U} \nabla_{*} \cdot\left(\tilde{\mu} \nabla_{*} u_{*}\right)+\left(u_{*}, \nabla_{*}\right) u_{*}+\frac{\mu d K^{-1}}{\rho U} u_{*}+\nabla_{*} p_{*}=\frac{d g}{U^{2}} f_{*} \\
\Rightarrow \tilde{s} \frac{\partial u_{*}}{\partial t_{*}}-\frac{1}{R e} \nabla_{*} \cdot\left(\tilde{\mu} \nabla_{*} u_{*}\right)+\left(u_{*}, \nabla_{*}\right) u_{*}+\frac{1}{R e D a} u_{*}+\nabla_{*} p_{*}=\frac{1}{F_{r}^{2}} f_{*}
\end{gathered}
$$

Accordingly the dimensionless form of Navier-Stokes-Brinkman system can be written as

$$
\begin{gathered}
\tilde{s} \frac{\partial u}{\partial t}+(u \cdot \nabla) u=-\nabla p+\frac{1}{R e} \nabla \cdot(\tilde{\mu} \nabla u)-\frac{1}{R e D a} u+\frac{1}{F_{r}^{2}} \tilde{f} \\
\nabla \cdot u=0
\end{gathered}
$$

where $\tilde{\mu}=1, \tilde{s}=1, \tilde{f}=f_{N S}, K^{-1}=0$ for the pure liquid region(Region 1$)$ and $\tilde{\mu}=\frac{\mu_{e f f}}{\mu}$, $\tilde{s}=s, \tilde{f}=f_{B}, K^{-1}=\epsilon_{p} k^{-1}$ for porous region (Region 2). The dimensionless parameters: Reyonald's, Darcy's and Froude numbers are given by $R e=\frac{U d}{\nu}, D a=\frac{\tilde{k}}{d^{2}}$ and $F_{r}=\frac{U}{\sqrt{g d}}$ respectively with $\tilde{k}=\frac{k}{\epsilon_{p}}$. The external body force $\tilde{f}=g e_{g}$, g is gravitational acceleration and $e_{g}$ is unit vector pointed in the direction of the gravitational force. If the porosity $\epsilon_{p}$ and the stone size $d_{50}$ of the rubble structure are known, then intrinsic permeability $k$ (with dimension $L^{2}$, L: length) can be determined as follows

$$
k=1.643 \times 10^{-7}\left(\frac{d_{50}}{d_{0}}\right)^{1.57} \frac{\epsilon_{p}^{3}}{\left(1-\epsilon_{p}\right)^{2}}
$$

where $d_{0}=10 \mathrm{~mm}$. To determine the inertial coefficient $s$, the added mass coefficient $c_{m}$ of the porous medium must be known. However, while $c_{m}$ is usually known for isolated simple shapes, it is generally unknown for random, densely packed material. In this study, the inertia coefficient $s$ was taken as 1 .

\subsection{Interface conditions between pure liquid and porous media}


In this section, we discuss some known approaches to treat the coupled problem between regions 1 and 2. Depending on the macroscopic model for the porous media flow, different interface conditions have to be specified on the interface. There are four interfaces at the submerged porous breakwater. The bottom interface is impermeable, while the other sides of the porous breakwater are permeable. A common choice of interface boundary conditions between Navier-Stokes and Brinkman equation is to assume continuous velocity and continuous normal component of the stress tensor. For continuity of velocity at interface

$$
\begin{aligned}
& \left.u\right|_{S_{f}}=\left.\epsilon_{p} u\right|_{S_{p}} \\
& \left.v\right|_{S_{f}}=\left.\epsilon_{p} v\right|_{S_{p}}
\end{aligned}
$$

Note that for impermeable structure, $\epsilon_{p}=0$, equations (12) and (13) are reduced to the no-slip boundary condition. The normal component of the stress tensor (continuity of normal component of stress tensor) at the interface is given by

$$
\left.n_{p} \cdot\left(\mu_{e f f} \nabla \mathbf{u}-p I\right)\right|_{S_{p}}=\left.n_{f} \cdot(\mu \nabla \mathbf{u}-p I)\right|_{S_{f}}
$$

where $S_{p}$ and $S_{f}$ are the interface of porous and interface of fluid parts respectively. Equation (14) can be written in dimensionless form as

$$
\left.n_{f} \cdot\left(\frac{1}{R e} \nabla \mathbf{u}-p I\right)\right|_{S_{f}}=\left.n_{p} \cdot\left(\frac{\mu_{e f f}}{\mu} \frac{1}{R e} \nabla \mathbf{u}-p I\right)\right|_{S_{p}}
$$

\subsection{Boundary Conditions}

To close the initial boundary value problem, appropriate initial conditions and boundary conditions are required. In this physical problem, there are four boundaries, such as: inlet, outlet, free surface and solid bed boundaries.

\subsubsection{Along solid wall}

The solid bed is assumed impermeable and no slip condition namely, $u=v=0$, is used. Thus on a solid wall both velocity components are set to zero at the bottom of the channel. In this paper we consider a solid wall parallel to the $x$-direction.

\subsubsection{Inlet}

The analytical solution for a Stokesian wave is imposed in the inlet boundary, thus the water elevation $\eta$ is given by

$$
\eta=d+\frac{H}{2} \cos (k x-\omega t)+\frac{\pi H^{2}}{8 L} \frac{\cosh (k d)}{\sinh ^{3}(k d)}(2+\cosh (2 k x-2 \omega t)
$$


where the wave number $k=\frac{2 \pi}{L}$ and wave frequency $\omega=\frac{2 \pi}{T}$. Inlet boundary conditions for velocity components are given as

$$
\begin{aligned}
& u=\frac{H}{2} \frac{g T}{L} \frac{\cosh (k y)}{\cosh (k d)} \cos (k x-\omega t)+\frac{3}{4}\left(\frac{\pi H}{L}\right)^{2} c \frac{\cosh (2 k y)}{\sinh ^{4}(k d)} \cos (2 k x-2 \omega t) \\
& v=\frac{H}{2} \frac{g T}{L} \frac{\sinh (k y)}{\cosh (k d)} \sin (k x-\omega t)+\frac{3}{4}\left(\frac{\pi H}{L}\right)^{2} c \frac{\sinh (2 k y)}{\sinh ^{4}(k d)} \sin (2 k x-2 \omega t)
\end{aligned}
$$

\subsubsection{Outlet}

The sommerfeld radiation condition is employed in the outlet boundary to avoid the reflection from the outlet boundary. If the outlet is selected far away from geometrical disturbances, the flow often reaches a fully developed state where no change occurs in the flow directions. Based on the wave equation and continuity equation, one can set

$$
\begin{aligned}
& \frac{\partial u}{\partial x}+c \frac{\partial v}{\partial y}=0 \\
& \frac{\partial \phi}{\partial t}+c \frac{\partial \phi}{\partial x}=0
\end{aligned}
$$

where $\phi$ is a physical variable such as pressure, velocity in the flow field and $c$ is the phase speed of the wave. For solitary wave $c=\sqrt{g\left(H_{i}+d\right)}$. In the numerical computation, when the wave passes over a porous Breakwater, the wave height at the downstream boundary is unknown a priori. Hence, this condition becomes unpractical. In this case, we simply extend the computation domain long enough such that the wave does not reach the downstream boundary of the domain.

\subsubsection{Free surface}

The type of flow which is subject to this paper contains a separation of the fluid with air. The separation surface is called the free surface. Boundary conditions on a free surface must satisfy kinematics and dynamic conditions. The kinematic boundary condition for free surface assumes that all fluid particles at the free surface must remain on the free surface and mathematically formulate as

$$
\frac{D \eta}{D t}=\frac{\partial \eta}{\partial t}+u \cdot \nabla \eta=v
$$

where $\eta$ denotes the free surface elevation. In terms of the dynamic boundary condition on free surface, stresses on a free surface must be balanced in normal and tangential conditions. The normal stress is equal to the atmospheric pressure and the tangential stress is zero. We ignore surface tension in the dynamic boundary conditions. Hence, the dynamic boundary condition in the normal direction was expressed by Huang [8] as

$$
p=\frac{\eta}{F_{r}^{2}}+\frac{2\left(1+\left(\frac{\partial \eta}{\partial x}\right)^{2}\right)}{R e\left(1-\left(\frac{\partial \eta}{\partial x}\right)^{2}\right)} \frac{\partial v}{\partial y}
$$


Further, the dynamics boundary condition in the tangential direction can be presented as

$$
\frac{\partial u}{\partial y}=\frac{-\partial v}{\partial x}+\frac{4}{\left(\frac{\partial \eta}{\partial x}\right)^{2}-1} \frac{\partial v}{\partial y} \frac{\partial \eta}{\partial x}
$$

where $p=p(x, \eta)$ is the hydrodynamic pressure at the free surface. In numerical computations, equation (22) is used to determine the pressure at the free surface and equation (23) is used to extrapolate the horizontal velocity at the free surface from the flow domain. The vertical velocity component is then calculated from the continuity equation using the known velocity component $u$, obtained from equation (23).

\subsection{Initial conditions}

In addition, we need initial conditions to start the numerical simulation and obtain a unique solution. There are three physical variables, $u, v$ and $p$ in the initial-boundary problem. Initial values for these variables have to be given. The initial flow field is assumed to be still, so velocity components are both zero throughout the flow field. The hydrostatic pressure is used to initialized the pressure field. The hydrodynamic pressure and surface displacements are also set to zero at time $t=0$.

\section{Numerical scheme}

The algorithm is based on a finite volume discretization on a non-uniform staggered Cartesian grid. This grid arrangement provides stronger coupling between the pressure and velocity variables. According to the staggered grid approach, velocity components are located at the middle points of the control surfaces, while pressures are defined at center of a computational cell, as shown in Figure 2. Using a staggered grid one obtains a control volume for pressure and four control volumes for velocities.

The control volumes are supposed to contain only a single phase either solid or fluid or porous that means interfaces between different media appear only at the interface of the control volumes.

\subsection{Discretization for continuity equation}

To discretize the continuity equation $\nabla \cdot \mathbf{u}=0$, the expression $\nabla \cdot \mathbf{u}$ is integrated on each of the control volume $\mathrm{V}$ for velocities and,then apply Gauss divergence theorem so as to get

$$
\int_{V} \nabla \cdot \mathbf{u} d V=0 \Rightarrow \int_{\partial V} \mathbf{u} \cdot n d s=0
$$

where $\partial V$ indicates the contour of the $E F G H$ or $(i, j)$ cell and $d s$ is the small change in the control surface with $\mathrm{n}$ being denoted as a unit outward normal vector to the surface. For the scalar control volumes centered at $(i, j)$, the discretized form of the above integral 


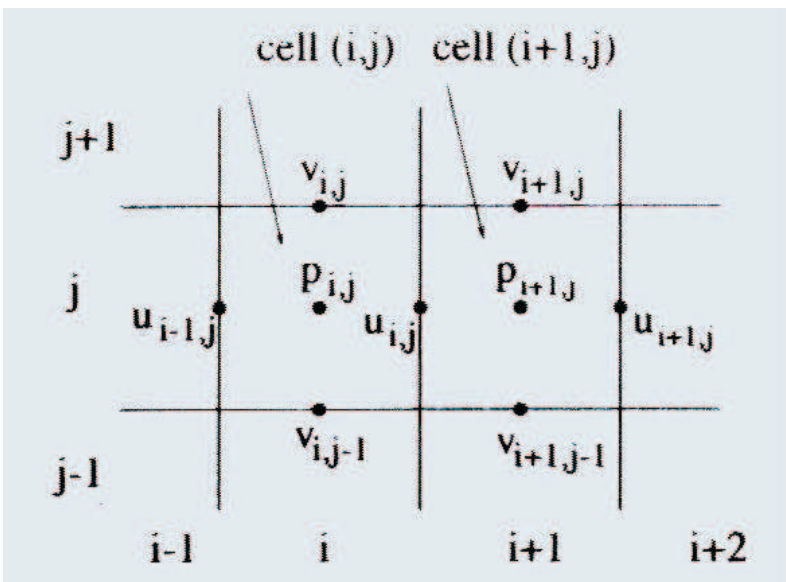

Figure 2: Staggered grid arrangement

is given by

$$
\int_{\partial V} \mathbf{u} \cdot n d s=\left(u_{i, j}-u_{i-1, j}\right) l_{j}+\left(v_{i, j}-v_{i, j-1}\right) h_{i}
$$

\subsection{Discretization for momentum equations}

To obtain finite volume method formulation, the governing momentum equations are integrated over the control volumes. Then, the integral form of $u$ and $v$ components of momentum equations are written as

$\int_{V} \frac{\partial u}{\partial t} d V=-\int_{\partial V} u(\mathbf{u} \cdot n) d s-\int_{V} \frac{1}{R e D a} u d V+\int_{\partial V}\left(\frac{\tilde{\mu}}{R e} \nabla u \cdot n\right) d s-\int_{\partial V} p_{x} \cdot n d s+\int_{V} \frac{1}{F_{r}^{2}} \tilde{f}_{x} d V$

$\int_{V} \frac{\partial v}{\partial t} d V=-\int_{\partial V} v(\mathbf{u} \cdot n) d s-\int_{V} \frac{1}{R e D a} v d V+\int_{\partial V}\left(\frac{\tilde{\mu}}{R e} \nabla v \cdot n\right) d s-\int_{\partial V} p_{y} \cdot n d s+\int_{V} \frac{1}{F_{r}^{2}} \tilde{f}_{y} d V$

Equations (25) and (26) can be rewritten as

$$
\begin{aligned}
& \int_{V}\left(\frac{\partial u}{\partial t}+\frac{1}{R e D a} u-\frac{1}{F_{r}^{2}} \tilde{f}_{x}\right) d V-\int_{\partial V}\left(\frac{\tilde{\mu}}{R e} \nabla u-u \mathbf{u}-p e_{1}\right) \cdot n d s=0 \\
& \int_{V}\left(\frac{\partial v}{\partial t}+\frac{1}{R e D a} v-\frac{1}{F_{r}^{2}} \tilde{f}_{y}\right) d V-\int_{\partial V}\left(\frac{\tilde{\mu}}{R e} \nabla v-v \mathbf{u}-p e_{2}\right) \cdot n d s=0 .
\end{aligned}
$$

\subsubsection{Discretization of volume integral}




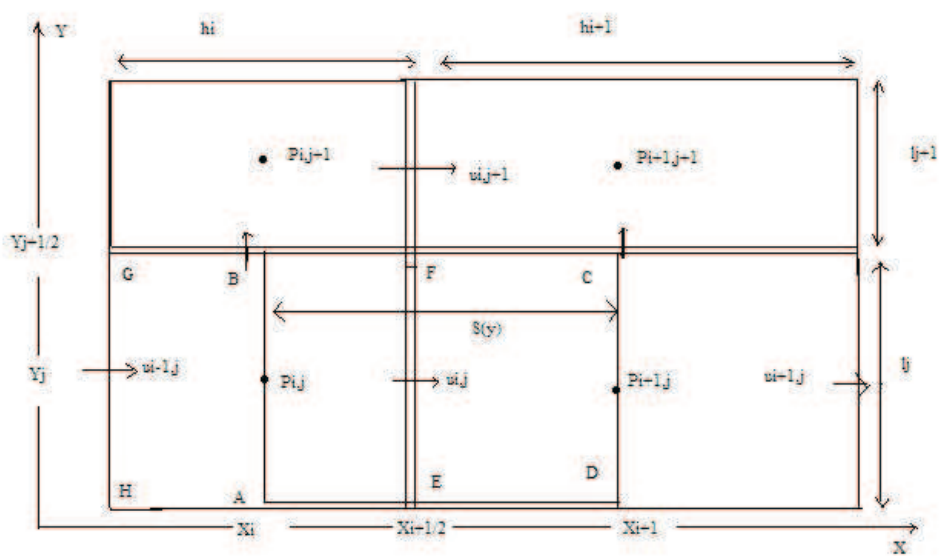

(a) A Cartesian staggered grid system on the $\mathrm{x}-\mathrm{y}$ plane

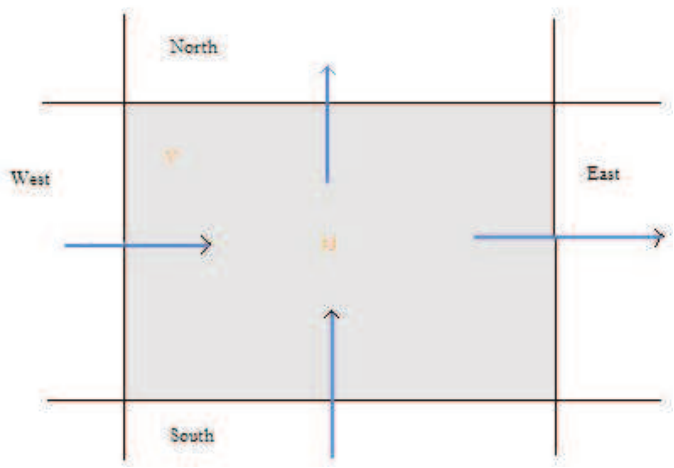

(b) Cell used for constructing a volume of fluid approximation

Figure 3: Computational cells

Without loss of generality consider the x-component of momentum equation. Integrating $\frac{\partial u}{\partial t}+\frac{\tilde{k}}{R e D a} u-\frac{1}{F_{r}^{2}} \tilde{f}_{x}$ over the control volume $A B C D$

$$
\begin{aligned}
\int_{A B C D}\left(\frac{\partial u}{\partial t}+\frac{1}{R e D a} u-\frac{1}{F_{r}^{2}} \tilde{f}_{x}\right) d V & =\left(\frac{\partial u}{\partial t}+\frac{1}{R e D a} u-\frac{1}{F_{r}^{2}} \tilde{f}_{x}\right) \cdot \Delta V \\
& =\left(\frac{u_{i, j}^{n+1}-u_{i, j}^{n}}{\delta t}+\frac{1}{\operatorname{ReDa}} u_{i, j}-\frac{1}{F_{r}^{2}} \tilde{f}_{x}\right) \cdot \Delta V \\
\int_{A B C D}\left(\frac{\partial u}{\partial t}+\frac{1}{R e D a} u-\frac{1}{F_{r}^{2}} \tilde{f}_{x}\right) d V & =\left(\frac{u_{i, j}^{n+1}-u_{i, j}^{n}}{\delta t}+\frac{1}{R e D a} u_{i, j}-\frac{1}{F_{r}^{2}} \tilde{f}_{x}\right) \cdot \Delta V
\end{aligned}
$$


where $\Delta V$ is the volume of control volume $A B C D$ and $\delta t$ is time subinterval.

\subsubsection{Discretization of surface integral}

To obtain a discretized form for surface integral, we have to integrate over the four faces of control volume $A B C D$ of figure 3 . Let $w_{1}=\frac{\tilde{\mu}}{R e} \nabla u-u \mathbf{u}-p e_{1}$, then we obtain

$$
\int_{\partial A B C D} w_{1} \cdot n d s=\int_{A B} w_{1} \cdot n d s+\int_{B C} w_{1} \cdot n d s+\int_{C D} w_{1} \cdot n d s+\int_{D A} w_{1} \cdot n d s
$$

We approximate the given integral over each phase of control volume $A B C D$. Along the phase $C D$ we have $\left.w_{1} \cdot n\right|_{C D}=\left.w_{1} \cdot e_{1}\right|_{C D}=\frac{\tilde{\mu}}{R e} \frac{\partial u}{\partial x}-[u]^{2}-p$. The segment $C D$ is inside $p_{i+1, j}$ th control volume, where the solution is assumed to be smooth, $w_{1} \cdot n$ is continues on $C D$ and we can use central point approximation for the integral,

$$
\left.\int_{C D} w_{1} \cdot n d s \approx w_{1} \cdot n\right|_{\left(x_{i+1}, y_{j}\right)} \cdot|C D| \approx\left(\frac{\tilde{\mu}}{R e} \frac{u_{i+1, j}-u_{i, j}}{h_{i+1}}-\left[u\left(x_{i+1}, y_{j}\right)\right]^{2}-p_{i+1, j}\right)|C D|
$$

where $|C D|$ is the length of edge $C D$. In order to handle the flow with high Reynolds number we use the so-called upwind difference scheme for the approximation of nonlinear convective terms. Accordingly using upwind scheme approximation for convective non linear term, equation (32) becomes

$$
\int_{C D} w_{1} \cdot n d s \approx\left(\frac{\tilde{\mu}}{R e} \frac{u_{i+1, j}-u_{i, j}}{h_{i+1}}-\tilde{u}_{i+1 / 2, j} u_{i, j}-p_{i+1, j}\right)|C D|
$$

Similarly, for $A B$,

$$
\int_{A B} w_{1} . n d s \approx-\left(\frac{\tilde{\mu}}{R e} \frac{u_{i, j}-u_{i-1, j}}{h_{i}}-\tilde{u}_{i-1 / 2, j} u_{i-1, j}-p_{i, j}\right)|A B|
$$

Along the phase $\mathrm{BC}$ we have $\left.w_{1} \cdot n\right|_{B C}=\left.w_{1} \cdot e_{2}\right|_{B C}=\frac{\tilde{\mu}}{R e} \frac{\partial u}{\partial y}-u v$. Thus we can approximate the volume integral along the edge $B C$ as

$$
\int_{B C} w_{1} \cdot n d s \approx \int_{S(y)} \frac{\tilde{\mu}}{R e} \frac{\partial u}{\partial y} d x-(u v)\left(x_{i+\frac{1}{2}}, y_{j+\frac{1}{2}}\right)|B C|
$$

where $S(y)=\left\{(x, y): x \in\left[x_{i}, x_{i+1}\right]\right\}$ and $\tilde{u}_{i+1 / 2, j}$ is priory known predictions for the values of $u_{i+1 / 2, j}$. Let us define an auxiliary function $w(y)$, where $y \in\left[y_{j}, y_{j+1}\right]$ such that $w(y)=\int_{S(y)} \frac{\tilde{\mu}}{R e} \frac{\partial u}{\partial y} d x$. We assume $u$ to be enough smooth and $u_{y}$ is continues along the interface and $w(y) \approx u_{y}\left(x_{i+\frac{1}{2}}, y\right) \int_{S(y)} \frac{\tilde{\mu}}{R e}$. Then $u_{i, j+1}-u_{i, j}=\int_{y_{j}}^{y_{j+1}} u_{y}\left(x_{i+\frac{1}{2}}, y\right) d y \approx$ $w\left(y_{j+\frac{1}{2}}\right) \int_{y_{j}}^{y_{j}+1} \frac{1}{\int_{S(y)} \frac{\mu}{R e} d x} d y$, If in each control volume $V$, the effective viscosities $\tilde{\mu}$ are 
constants and equal to $\tilde{\mu}_{i, j}$ then it is easy to calculate the integral in the expressions for the coefficients. for $y \in\left(y_{j}, y_{j+\frac{1}{2}}\right)$

$$
\int_{S(y)} \tilde{\mu} d x=\frac{1}{2}\left(\tilde{\mu}_{i, j} h_{i}+\tilde{\mu}_{i+1, j} h_{i+1}\right)
$$

if $y \in\left(y_{j+\frac{1}{2}}, y_{j+1}\right)$

$$
\begin{aligned}
\int_{S(y)} \tilde{\mu} d x= & \frac{1}{2}\left(\tilde{\mu}_{i, j+1} h_{i}+\tilde{\mu}_{i+1, j+1} h_{i+1}\right) \\
\Rightarrow u_{i, j+1}-u_{i, j} \approx & w\left(y_{j+\frac{1}{2}}\right)\left(\int_{y_{j}}^{y_{j+\frac{1}{2}}} \frac{R e}{\frac{1}{2}\left(\tilde{\mu}_{i, j} h_{i}+\tilde{\mu}_{i+1, j} h_{i+1}\right)} d y\right. \\
& \left.+\int_{y_{j+\frac{1}{2}}}^{y_{j+1}} \frac{R e}{\frac{1}{2}\left(\tilde{\mu}_{i, j+1} h_{i}+\tilde{\mu}_{i+1, j+1} h_{i+1}\right)} d y\right) \\
\approx & w\left(y_{j+\frac{1}{2}}\right)\left(\frac{l_{j} R e}{\tilde{\mu}_{i, j} h_{i}+\tilde{\mu}_{i+1, j} h_{i+1}}+\frac{l_{j+1} R e}{\tilde{\mu}_{i, j+1} h_{i}+\tilde{\mu}_{i+1, j+1} h_{i+1}}\right)
\end{aligned}
$$

Let $C_{i, j+\frac{1}{2}}=\operatorname{Re}\left(\frac{l_{j}}{\tilde{\mu}_{i, j} h_{i}+\tilde{\mu}_{i+1, j} h_{i+1}}+\frac{l_{j+1}}{\tilde{\mu}_{i, j+1} h_{i}+\tilde{\mu}_{i+1, j+1} h_{i+1}}\right)$, then

$$
\begin{gathered}
w\left(y_{j+\frac{1}{2}}\right)=\frac{u_{i, j+1}-u_{i, j}}{C_{i, j+\frac{1}{2}}} \\
\int_{B C} w_{1} \cdot n d s \approx w\left(y_{j+\frac{1}{2}}\right)-(u v)\left(x_{i+\frac{1}{2}}, y_{j+\frac{1}{2}}\right)|B C| \\
\approx \frac{u_{i, j+1}-u_{i, j}}{C_{i, j+\frac{1}{2}}}-(u v)\left(x_{i+\frac{1}{2}}, y_{j+\frac{1}{2}}\right)|B C|
\end{gathered}
$$

Again applying upwind scheme approximation for non linear term, the final expression for the integral over the phase $B C$ becomes

$$
\int_{B C} w_{1} \cdot n d s \approx \frac{u_{i, j+1}-u_{i, j}}{C_{i, j+\frac{1}{2}}}-\tilde{v}_{i+\frac{1}{2}, j}\left(u_{i, j}-\tilde{u}_{i, j}\right)|B C|
$$

Similarly, for DA

$$
\begin{aligned}
\int_{D A} w_{1} \cdot n d s & \approx-w\left(y_{j-\frac{1}{2}}\right)-(u v)\left(x_{i+\frac{1}{2}}, y_{j-\frac{1}{2}}\right)|D A| \\
& \approx \frac{-\left(u_{i, j}-u_{i, j-1}\right)}{C_{i, j-\frac{1}{2}}}-(u v)\left(x_{i+\frac{1}{2}}, y_{j-\frac{1}{2}}\right)|D A| \\
& \approx \frac{-\left(u_{i, j}-u_{i, j-1}\right)}{C_{i, j-\frac{1}{2}}}-\tilde{v}_{i+\frac{1}{2}, j-1}\left(u_{i, j-1}-\tilde{u}_{i, j-1}\right)|D A|
\end{aligned}
$$


where

$$
C_{i, j-\frac{1}{2}}=\operatorname{Re}\left(\frac{l_{j-1}}{\tilde{\mu}_{i, j-1} h_{i}+\tilde{\mu}_{i+1, j} h_{i+1}}+\frac{l_{j}}{\tilde{\mu}_{i, j} h_{i}+\tilde{\mu}_{i+1, j+1} h_{i+1}}\right)
$$

\subsection{The pressure Poisson equation}

The Navier-Stokes-Brinkman equations clearly determine the respective velocity components so that their roles are clearly defined. This leaves the continuity equation, which does not contain the pressure, to determine the pressure. To do this, the most common method is based on combining the two equations. Thus we can derive pressure Poisson equation by using projection method. Consider the following incompressible Navier-Stokes-Brinkman equation in Lagrangian form

$$
\begin{aligned}
& \frac{d x}{d t}=v \\
& \frac{d v}{d t}=\frac{\partial v}{\partial t}+(v \cdot \nabla v) \\
& \frac{d v}{d t}=-\nabla p-\frac{1}{R e D a} v+\frac{\tilde{\mu}}{R e} \Delta v+\frac{1}{F_{r}^{2}} \tilde{f}
\end{aligned}
$$

where $x=(x, y)$ and $\vec{v}=(u, v)$. Consider $\frac{d x}{d t}=v$, then

$$
\frac{x^{(n+1)}-x^{n}}{\delta t}=v^{(n)} \Rightarrow x^{(n+1)}=x^{(n)}+\delta t * v^{(n)}
$$

From equation (40) we also have

$$
\frac{v^{(n+1)}-v^{*}+v^{*}-v^{n}}{\delta t}=-\nabla p^{(n+1)}-\frac{1}{R e D a} v^{(n)}+\frac{\tilde{\mu}}{R e} \triangle v^{(n)}+\frac{1}{F_{r}^{2}} \tilde{f}^{(n)}
$$

using projection method

$$
\begin{gathered}
\frac{v^{(n+1)}-v^{*}}{\delta t}=-\nabla p^{(n+1)} \\
\frac{v^{*}-v^{(n)}}{\delta t}=-\frac{1}{R e D a} v^{(n)}+\frac{\tilde{\mu}}{R e} \Delta v^{(n)}+\frac{1}{F_{r}^{2}} \tilde{f}^{(n)}
\end{gathered}
$$

From $\frac{v^{(n+1)}-v^{*}}{\delta t}=-\nabla p^{(n+1)}$ we have $v^{(n+1)}=v^{*}-\delta t \cdot\left(\nabla p^{(n+1)}\right)$ apply divergence on both sides with the incompressibility constraint $\nabla \cdot v^{(n+1)}=0$, then we obtain

$$
\begin{gathered}
\nabla \cdot v^{(n+1)}=\nabla \cdot v^{*}-\delta t \cdot\left(\nabla \cdot \nabla p^{(n+1)}\right) \\
\Rightarrow \Delta p^{(n+1)}=\frac{\nabla \cdot v^{*}}{\delta t}
\end{gathered}
$$

where $v^{*}$ is an intermediate velocity ( the tentative velocity field ). The boundary condition for solid walls as well as for inflow boundaries is obtained by projecting equation 
$v^{(n+1)}=v^{*}-\delta t \cdot\left(\nabla p^{(n+1)}\right)$ on the outward unit normal vector $(\vec{n})$ to the boundary. Thus, we obtain the Neumann boundary condition , $\left(\frac{\partial p}{\partial \vec{n}}\right)^{n+1}=\frac{-1}{\delta t}\left(\vec{v}^{n+1}-\vec{v}^{n}\right) \cdot \vec{n}$, on the boundary. Assuming $\vec{v}^{n} \cdot \vec{n}=0$ on the boundary

$$
\frac{\partial p}{\partial \vec{n}}^{(n+1)}=0
$$

Moreover, Dirichlet boundary condition is applied for the free surface as well as for outflow particles in the context of the pressure Poisson equation (43). We note that particle positions change only in the first step. The intermediate velocity $v^{*}$ is obtained on the new particle positions. Finally, the pressure Poisson equation and the divergence free velocity vector are also computed on the same new particle positions.

\subsection{Numerical methods for free surface flows (Volume of Fluid Method)}

The most important property of the flow that is studied in this paper is the presence of a free surface. The flow is mainly determined by this free surface, therefore a proper discretization is required to capture its dynamics. To obtain numerical solutions of the governing equations, the whole computational domain is divided into Cartesian cells. The free surface calculation is a challenging point in the numerical study. An appropriate approach to treat free surface variation has to satisfy two requirements. First of all, it has to capture free surface profiles. The other point is to impose free surface boundary conditions at the captured free surface. We adopt the Volume -of- Fluid (VOF) method Hirt and Nichols [5] to evaluate the free surface variation. The main principle of VOF is to define another variable called the VOF function, $F(x, y, t)$. It is also defined at the center of computational cell. Its value varies from 0 to 1 . Due to a free surface, computational cell may contain fluids or not. A cell full of fluid is called a full cell. The VOF functions, in a full cell are defined as 1 . On the other hand, if a cell does not contain any fluid, it will be called an empty cell with $F$ defined as 0 for an empty cell. In addition, a cell containing a free surface is called a surface cell with $F$ varying between 0 and 1 for a surface cell;

$$
F(i, j)=\frac{\text { volume1 }}{\text { volume } 2}
$$

where volume 1 is volume of the fluid in a given cell and volume 2 is volume of the corresponding cell. $F=1$ means the cell is full of water, $F=0$ is air cell, while $0<F<1$ means the cell contains the free surface. Free surface boundary conditions have imposed at surface cells. The free surface is time dependent, so the VOF functions, $F$, has to be calculated at each time step and satisfies the convection equation,

$$
\frac{\partial F}{\partial t}+u \frac{\partial F}{\partial x}+v \frac{\partial F}{\partial y}=0
$$

For incompressible fluid flow equation (46) may be combined with continuity equation (2) to yield the equation

$$
\frac{\partial F}{\partial t}+\nabla \cdot(F \mathbf{u})=0
$$


where $\mathbf{u}=(u, v)$. In addition to equation (47), Hirt and Nichols [5] derived the donor acceptor method to solve the formula. Let a horizontal velocity component, u be located at the center of a control face. If $u$ is positive, then the cell in the left side of the control face is called a donor. On the other hand, the cell in the right hand side is called an acceptor. The variation of $\mathrm{F}$ is evaluated using the above given formula (47), which may be integrated in the control volume of the single $(i, j)$ cell

$$
\begin{aligned}
\int_{V} \frac{\partial F}{\partial t} d V+\int_{V} \nabla \cdot(\mathbf{u} F) d V=0 \\
\Rightarrow \int_{V} \frac{\partial F}{\partial t} d V+\int_{\partial V}(\mathbf{u} \cdot \vec{n}) F d s=0
\end{aligned}
$$

For the sides west and east the above integral yields $\frac{\Delta F}{\Delta t} \Delta V+F u l_{j}=0$, while for the sides south and north we get $\frac{\Delta F}{\Delta t} \Delta V+F v h_{i}=0$. In a strong convective flow from west to east, the central difference scheme treatment is unstable because the west face should receive much stronger influencing from west node $(W)$ than from central node $(P)$. Thus, the upwind differencing scheme or donor cell differencing scheme takes into account the flow direction when determining the value at a cell face. Let $\operatorname{sgn}(u)$ be the sign of velocity $u$, whether the flow is in the positive direction (west to east) or negative direction (east to west). Since $V=h_{i} l_{j}$, using first order upwind discretization we have

$$
\left.\Delta F_{i, j}\right|_{i+\frac{1}{2}, j}=-(1+\operatorname{sgn}(u)) \frac{u \cdot \delta t}{2 h_{i}} F_{i, j}+(1-\operatorname{sgn}(u)) \frac{u \cdot \delta t}{2 h_{i}} F_{i+1, j}
$$

where $\Delta F_{i+\frac{1}{2}, j}$ denotes the increment in $F_{i, j}$ due to the fluxes over the east phase and in which $u=u_{i+\frac{1}{2}, j}$. Similar expression can be derived for the change in $F_{i, j}$ due to the contribution of the other sides, yielding

$$
\left.\Delta F_{i, j}\right|_{i-\frac{1}{2}, j}=(1+\operatorname{sgn}(u)) \frac{u \cdot \delta t}{2 h_{i}} F_{i-1, j}-(1-\operatorname{sgn}(u)) \frac{u \cdot \delta t}{2 h_{i}} F_{i, j}
$$

This denotes the changes in $F$ due to the flux over the west phase and in which $u=$ $u_{i-\frac{1}{2}, j}$. For the cell $(i, j)$ evaluate at $\left(i, j+\frac{1}{2}\right)$ we also have an expression

$$
\left.\Delta F_{i, j}\right|_{i, j+\frac{1}{2}}=-(1+\operatorname{sgn}(v)) \frac{v \cdot \delta t}{2 l_{j}} F_{i, j}+(1-\operatorname{sgn}(v)) \frac{v \cdot \delta t}{2 l_{j}} F_{i, j+1}
$$

where $\Delta F_{i, j+\frac{1}{2}}$ denotes the increment in $F_{i, j}$ due to the fluxes over the north phase and in which $v=v_{i, j+\frac{1}{2}}$. For the change in $F_{i, j}$ due to the contribution of the south phase is also written as

$$
\left.\Delta F_{i, j}\right|_{i, j-\frac{1}{2}}=(1+\operatorname{sgn}(v)) \frac{v \cdot \delta t}{2 l_{j}} F_{i, j-1}-(1-\operatorname{sgn}(v)) \frac{v \cdot \delta t}{2 l_{j}} F_{i, j}
$$

Assembling the changes in $F$ from all four sides and updating $F$ yields the new distribution of the liquid in the computational domain. In order to ensure that the total amount 
of fluid does not change, i.e i.e $\sum_{i, j} F_{i, j}$ remains constant, the value of $F$ in the surface cells will be modified. After the new distribution of $\mathrm{F}$ has been calculated the labelling of empty, surface and full cells is performed again. The boundary conditions on the free surface will be set again, after which the following time step can be taken.

\subsection{The SIMPLE algorithm}

The acronym SIMPLE stands for Semi-Implicit Method for Pressure-Linked Equations. The algorithm was originally put forward by Patankar and Spalding and is essentially a guess and correct procedure for the calculation of pressure on the staggered grid arrangement introduce on Figure 2 above. SIMPLE algorithm is one of the fundamental algorithms for solving the resulting coupled system of algebraic equations. The method is iterative and when other scalars are coupled to the momentum equations, the calculation needs to be done sequentially [12]. We apply the sequence of operations in a CFD procedure which employs the SIMPLE algorithms in the following way

1. Guess initial values of pressure field $\left(p^{*}\right)^{n}$

2. Set initial value of velocity field $\left(u^{*}\right)^{n}$ and $\left(v^{*}\right)^{n}$

3. Solving for the intermediate (tentative) velocity fields $\left(u^{*}\right)^{n+1}$ and $\left(v^{*}\right)^{n+1}$

4. solving for the pressure correction $p^{c}$ from equation (43)

5. Solving for corrected pressure, using equation

$$
p^{n+1}=\left(p^{*}\right)^{n}+p^{c}
$$

6. Solving for velocity correction $u^{c}$ and $v^{c}$, projection of velocity to divergence free field

$$
\begin{aligned}
& u_{i+\frac{1}{2}, j}^{c}=-\frac{\delta t}{\frac{1}{2}\left(h_{i}+h_{i+1}\right)}\left(P_{i+1, j}^{c}-P_{i, j}^{c}\right) \\
& v_{i, j+\frac{1}{2}}^{c}=-\frac{\delta t}{\frac{1}{2}\left(l_{j}+l_{j+1}\right)}\left(P_{i, j+1}^{c}-P_{i, j}^{c}\right)
\end{aligned}
$$

7. Solving for corrected velocity

$$
\begin{aligned}
& u^{n+1}=\left(u^{*}\right)^{n}+u^{c} \\
& v^{n+1}=\left(v^{*}\right)^{n}+v^{c}
\end{aligned}
$$

8. Proceeding to a new time step. 


\section{Results and discussions}

In this section, we validate the proposed model, at first; the numerical solutions for a solitary wave are compared with previous studies. In addition, the established model is coupled with the porous structure and is used to simulate progressive waves over submerged porous breakwater. The effect of the Reynolds's and Darcy's numbers on the wave transformation, reflection and transmission for solitary wave is investigated subsequently; for free surface flow the proposed model is utilized to simulate the broken dam problem.

\subsection{Propagation of solitary waves in a numerical wave tank}

In this study, the incident solitary waves were generated using a numerical piston-type wave maker developed by Huang [8]. The attenuation of a solitary wave propagating in a channel with a flat bottom was determined using the present numerical scheme and compared with the analytic and experimental results. Russell [11] conducted experiments regarding waves in a shallow water tank. He observed wave heights of travelling solitary waves are affected by the fluid viscosity. After a century, Keulegan [9] reconsider the viscous solitary wave problem and obtained analytic solutions. According to Keulegan, the damping rate of the solitary wave is

$$
\left(\frac{H}{d}\right)^{\frac{-1}{4}}=\left(\frac{H_{i}}{d}\right)^{\frac{-1}{4}}+\frac{1}{12}\left(1+\frac{2 d}{\tilde{w}}\right) \sqrt{\frac{\nu}{d \sqrt{g d}}} \cdot \frac{x}{d}
$$

where $H_{i}$ is the initial wave height, $\nu$ is the kinematics viscosity of the fluid, $x$ is the distance travelled by the solitary wave, and $\tilde{w}$ is the width of the channel. Mei [10] rederived Keulegan's results using the perturbation method and gave the law of attenuation as

$$
H^{\frac{-1}{4}}=H_{i}^{\frac{-1}{4}}+0.08356 \sqrt{\frac{\nu}{d^{2} \sqrt{g}}} \cdot \frac{x}{d}
$$

This is indeed a special case of Eq.(58) as $\tilde{w}$ approaches infinity. Mei assumed that most of viscous effect come from the solid boundary and he obtained the relationship between wave height and time is

$$
H^{\frac{-1}{4}}=H_{i}^{\frac{-1}{4}}+\frac{0.08356}{\sqrt{\tilde{R} e}} t \sqrt{g d} \cdot d^{\frac{-5}{4}}
$$

where $\tilde{R} e=\frac{\sqrt{g d d}}{\nu}$ and $t$ is time. We consider numerical tank of length $20 \mathrm{~m}$ and depth $0.2 \mathrm{~m}$. The wave with height $0.075 \mathrm{~m}$ was propagated toward the right direction. The viscous solitary wave travels at Reynolds number 1000. The whole simulation time is 10s. Figure 4 shows that the evaluation of moving solitary wave. We learned from the figure, a solitary wave decays due to the energy dissipation caused by the viscosity. Accordingly due to the fluid viscosity, the wave height of moving solitary wave decreases as time goes. Figure 5 shows the time history of the wave height. The wave height 
decays due to fluid viscosity as the wave travels. It reveals that our numerical results agree very well with the analytic solution. The established numerical model simulates the viscous damping in the moving solitary wave successfully. On the other hand, the viscous effect does not only reduce the wave height but also slow down the wave velocity. Russell's experimental data for a solitary wave cited by Keulegan is also shown in Figure 6 to provide more information for comparison. The numerical values shown in Figure 6 where calculated with $, H_{i}=0.028, \nu=1.14 \times 10^{-6} \mathrm{~m}^{2} / \mathrm{s}, g=9.81 \mathrm{~m} / \mathrm{s}^{2}$ and $\mathrm{d}=0.2 \mathrm{~m}$, to be consistent with Russell's experimental conditions. We noted that our numerical results lie between the results calculated from equation (60) and Russell's experimental data.

\subsection{Numerical tests for free surface model}

One example has been chosen to illustrate the accuracy and capabilities of the VOF code. In this example, experimental information is available for comparison with the calculated results. This example offer a substantial challenge to any free boundary method.

\subsubsection{The broken dam problem}

This is a very popular and simple test case to validate many numerical schemes for the simulations of free surface flows. It consists of simple initial configurations and simple initial and boundary conditions. The experimental results are available in W.J. Moyce [14]. Consider a rectangular column of water with a width of $L=5.1 \mathrm{~m}$ and a height of $h=10.2 m$. The lines $x=0, y=0$ and $x=25$ consists of the solid wall. In the simulation, the upper and the right boundary of the water columns are consider as the free surface boundary. Initially, $100 \times 100$ grid points are considered. The gravity with $g=9.81 \frac{m}{s^{2}}$ acts downwards, the initial velocity is set to zero. The initial pressure $p_{0}$ is also considered to be zero. The surface tension forces are neglected. When the right wall(dam) is removed, the water column collapses under the influence of gravity. The dynamic viscosity of the fluid is $\mu=0.5 \frac{\mathrm{kg}}{\mathrm{ms}}$. No slip boundary condition is used on the solid walls. The particles, plotted successively in time, are shown in Figure 7. The numerical results can be compared with the experimental data given in W.J. Moyce [14]. In Figure 8, the position of the leading fluid front versus time and height of the water column versus time are compared. The figure shows a good agreement between the numerical and experimental result.

\subsection{Effects of structure permeability}

After having verified the accuracy of the numerical scheme, the interaction of a solitary wave and a submerged breakwater was studied. A schematic diagram of a solitary wave passing over a submerged breakwater is shown in Figure 9. The breakwater can be permeable or impermeable and for the sake of simplicity, its shape is taken to be rectangular. A piston-type wave maker with stroke $s_{0}$ is located at $x=0$ and generates the incident solitary waves. In Table 1, Case 1 to Case 3 (Type I) represents the reference 

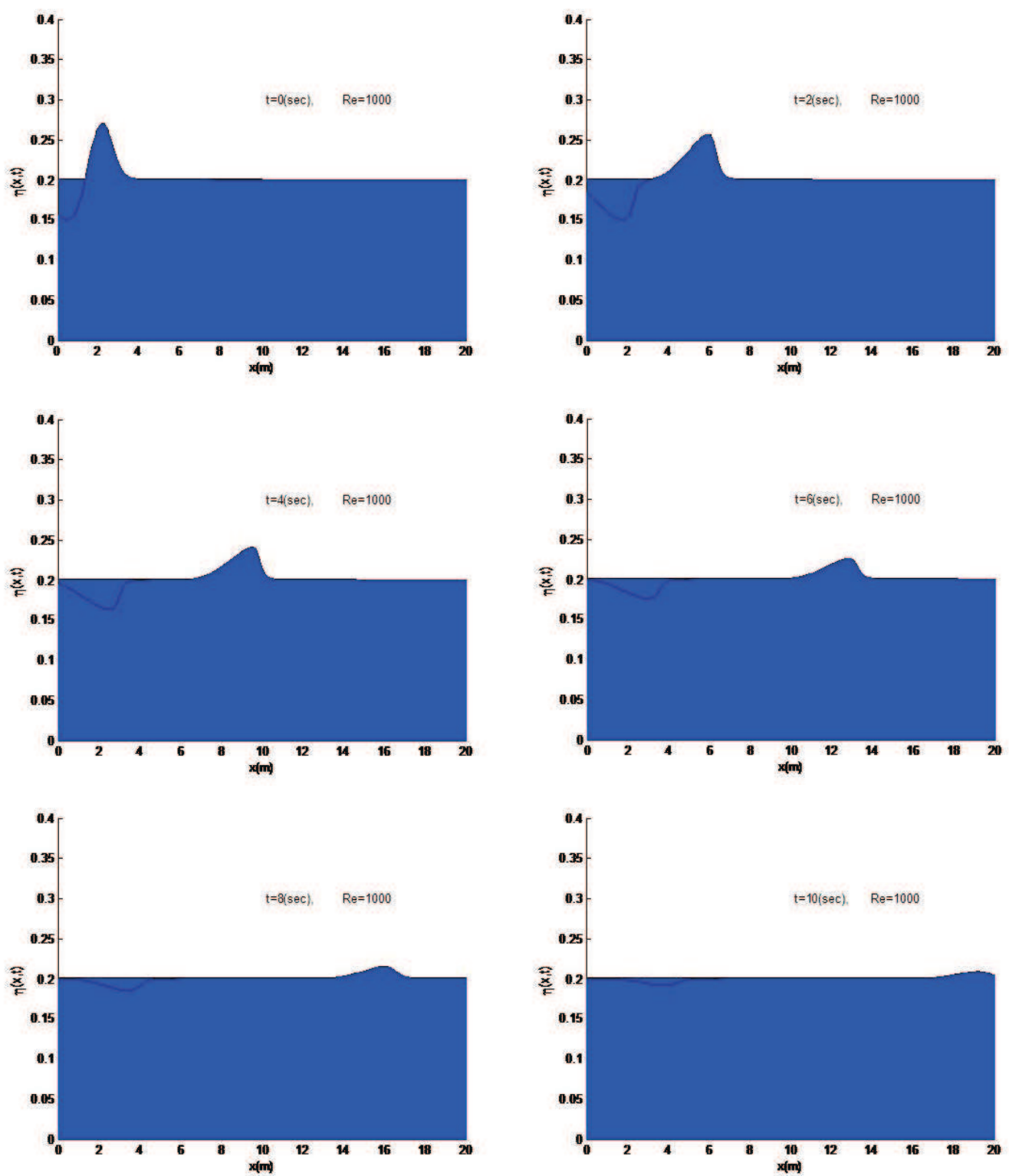

Figure 4: The evolution of a moving solitary wave with out considering porous breakwater.

values for $\frac{H_{i}}{d}, \frac{w}{d}$ and $\frac{h}{d}$ with the breakwater in Case 1 being impermeable, and permeable in Cases 2 and 3 with Darcy number $D a$ equals to $0.612 \times 10^{-6}$ and $2.741 \times 10^{-6}$ 


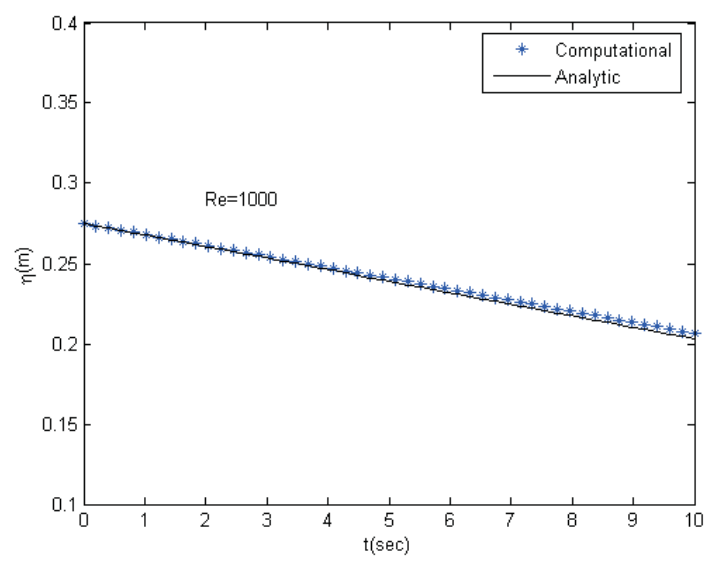

Figure 5: The wave height decays due to fluid viscosity as the wave travels

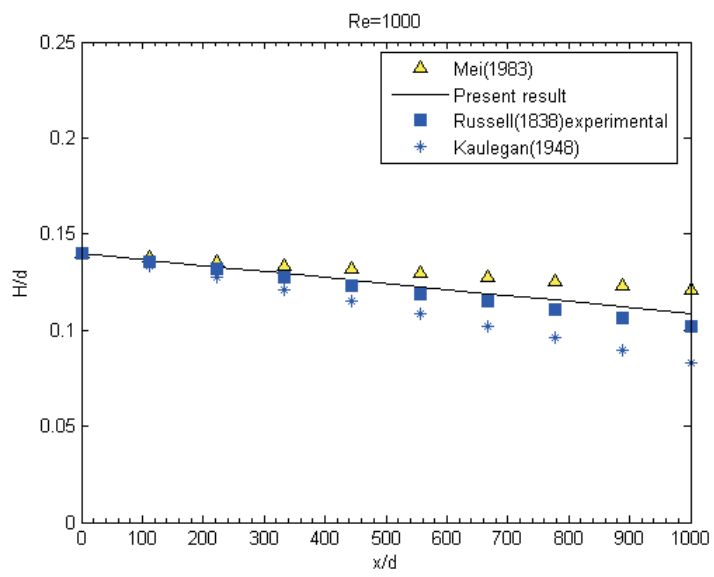

Figure 6: Attenuation of a solitary wave of $\mathrm{Hi}=0.04$ propagating in a channel with a flat bottom

respectively. In Cases 4 to 6 (Type II), the incident wave height $\frac{H_{i}}{d}$ increases from the reference value of 0.20 to 0.40 . In Cases 7 to 9 (Type III), the breakwater height $\frac{h}{d}$ increases from 0.5 to 0.75 .

In this section, we concentrate on the effect of the structure permeability on the wave transformation, reflection and transmission as a solitary wave passes over a submerged breakwater. The transformation of a solitary wave passing over a submerged breakwater for Cases 4 to 6 (Type II) with $\frac{w}{d}=1$, the breakwater width is much smaller than the wavelength. Thus, the nonlinear effect caused by the breakwater is minor, hence there 

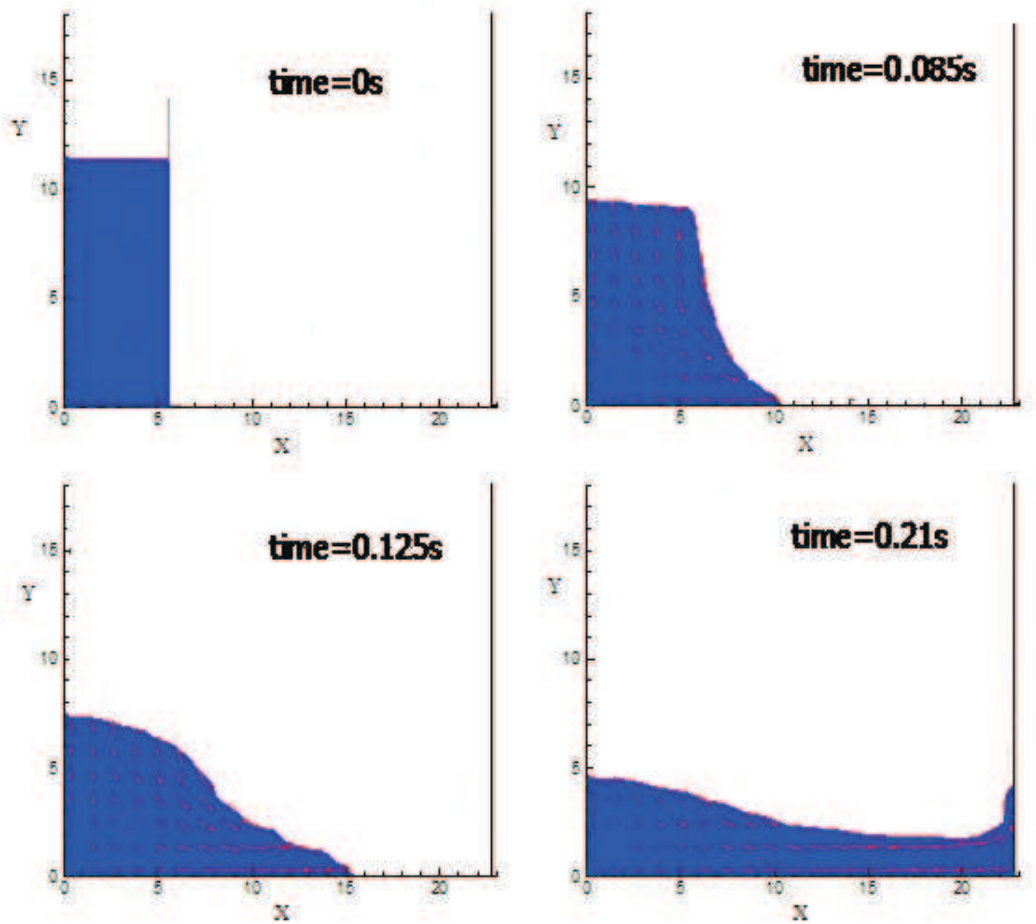

Figure 7: Velocity vectors and fluid configuration for the broken dam at times $t=0 \mathrm{~s}$, $t=0.085 s, t=0.125 s$ and $t=0.21 s$

Table 1: Numerical condition of the incident solitary wave and the breakwater width and height.

\begin{tabular}{cccccc}
\hline Type & case & $H_{i} / d$ & $w / d$ & $h / d$ & $D a\left(\times 10^{-6}\right)$ \\
\hline I & 1 & 0.2 & 1.0 & 0.5 & - \\
& 2 & 0.2 & 1.0 & 0.5 & 0.612 \\
II & 3 & 0.2 & 1.0 & 0.5 & 2.741 \\
& 4 & 0.4 & 1.0 & 0.5 & - \\
& 5 & 0.4 & 1.0 & 0.5 & 0.612 \\
III & 6 & 0.4 & 1.0 & 0.5 & 2.741 \\
& 7 & 0.2 & 1.0 & 0.75 & - \\
& 8 & 0.2 & 1.0 & 0.75 & 0.612 \\
IV & 9 & 0.2 & 1.0 & 0.75 & 2.741 \\
& 10 & 0.2 & 25.0 & 0.5 & - \\
& 11 & 0.2 & 25.0 & 0.5 & 0.612 \\
& 12 & 0.2 & 25.0 & 0.5 & 2.741 \\
\hline
\end{tabular}




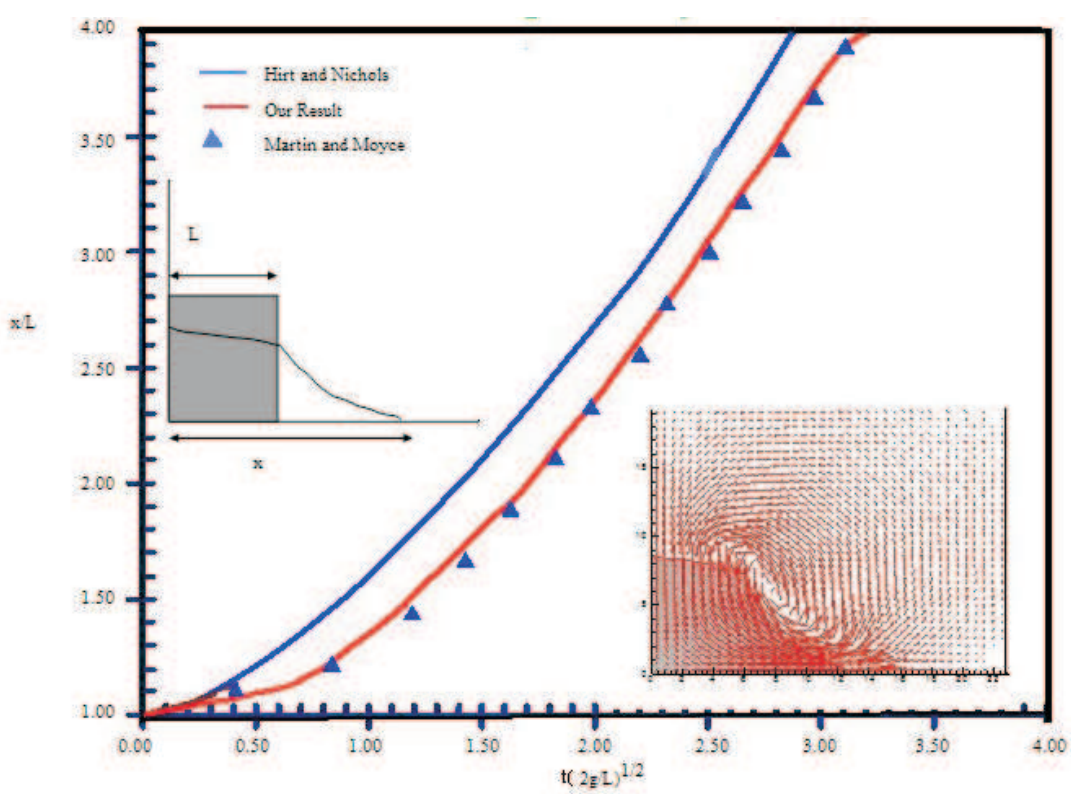

Figure 8: Time history of leading edge of the wave for comparison of present numerical result with experimental data

is no apparent wave distortion noticed. The effect of the structure permeability is not distinguishable either. As the breakwater width increases from 1.0 to 25.0 in cases 10 to 12 (Type IV), the permeability effect becomes obvious. The transmitted wave height decreases as the Darcy number $D a$ increases from $0.612 \times 10^{-6}$ to $2.741 \times 10^{-6}$. To see how the wave height varies as a solitary wave passes over a breakwater, Figure 10 shows the maximum value of the water elevation at different locations in the wave tank for Cases 1 to 12, categorized into Type I to Type IV, the rectangle indicates the location of the breakwater. The rise of the wave height near the leading edge of the breakwater is due to the shallow effect. Consequently, the wave height decreases as part of the wave is reflected. For the small width breakwaters, Figure 10(A) to (C), the wave height above the breakwater decreases slightly due to the energy dissipation caused by the wave and structure interaction and reaches a minimum value at the trailing edge. Afterwards, the wave height increases a little, as the reflected wave is generated. Its clearly shown at Figure 10(D) that for wide impermeable breakwater, due to the nonlinear effect, the wave height above the breakwater increases continually, which may eventually result in wave breaking. However, a wide permeable breakwater will reduce the wave height due to the additional energy dissipation caused by the flow friction within the porous structure. As the Darcy number increases from $0.612 \times 10^{-6}$ to $2.741 \times 10^{-6}$, the transmitted wave height decreases. However, if $D a$ is further increased from $1.119 \times 10^{-5}$ to $2.225 \times 10^{-5}$, the transmitted wave height increases. Regarding the transmission coefficient for the porous 


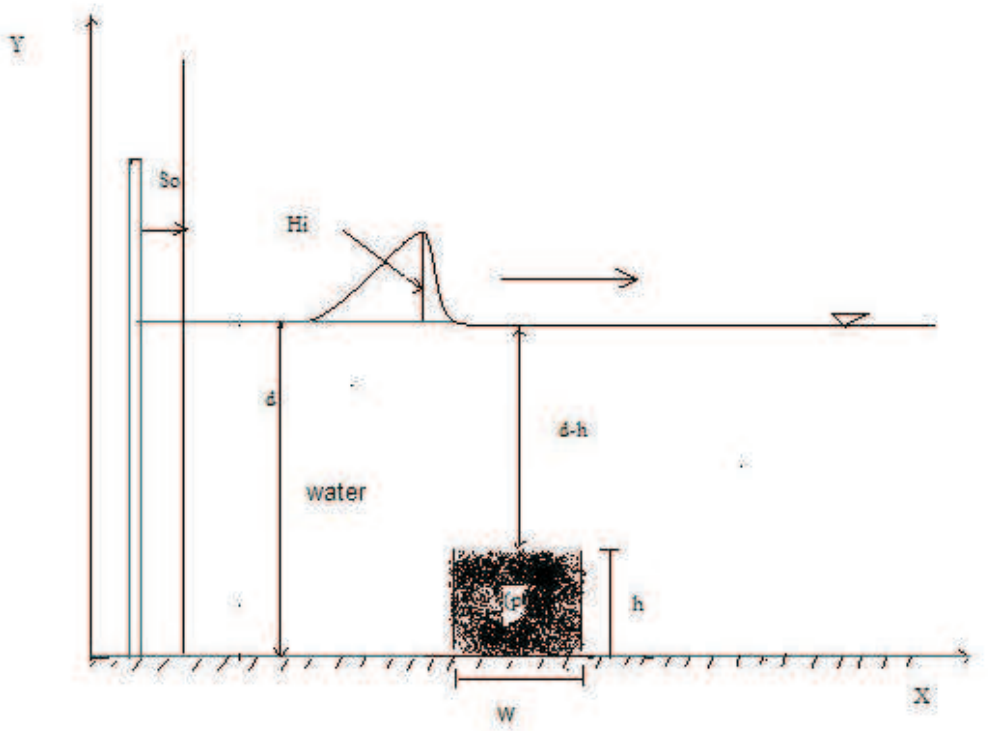

Figure 9: Schematic diagram of a solitary wave propagating over a submerged porous breakwater

Table 2: Numerical condition of the incident solitary wave and the breakwater width and height.

\begin{tabular}{cccccc}
\hline Type & case & $H_{i} / d$ & $w / d$ & $h / d$ & $D a\left(\times 10^{-6}\right)$ \\
\hline 13 & 0.2 & 10.0 & 0.5 & - \\
& 14 & 0.2 & 10.0 & 0.5 & 0.612 \\
& 15 & 0.2 & 10.0 & 0.5 & 2.741 \\
& 16 & 0.2 & 15.0 & 0.5 & - \\
$\mathrm{V}$ & 17 & 0.2 & 15.0 & 0.5 & 0.612 \\
& 18 & 0.2 & 15.0 & 0.5 & 2.741 \\
& 19 & 0.2 & 20.0 & 0.8 & - \\
& 20 & 0.2 & 20.0 & 0.8 & 0.612 \\
& 21 & 0.2 & 20.0 & 0.8 & 2.741 \\
& 22 & 0.2 & 25.0 & 0.5 & - \\
23 & 0.2 & 25.0 & 0.5 & 0.612 \\
& 24 & 0.2 & 25.0 & 0.5 & 2.741 \\
& 25 & 0.2 & 30.0 & 0.5 & - \\
26 & 0.2 & 30.0 & 0.5 & 0.612 \\
27 & 0.2 & 30.0 & 0.5 & 2.741 \\
\hline
\end{tabular}



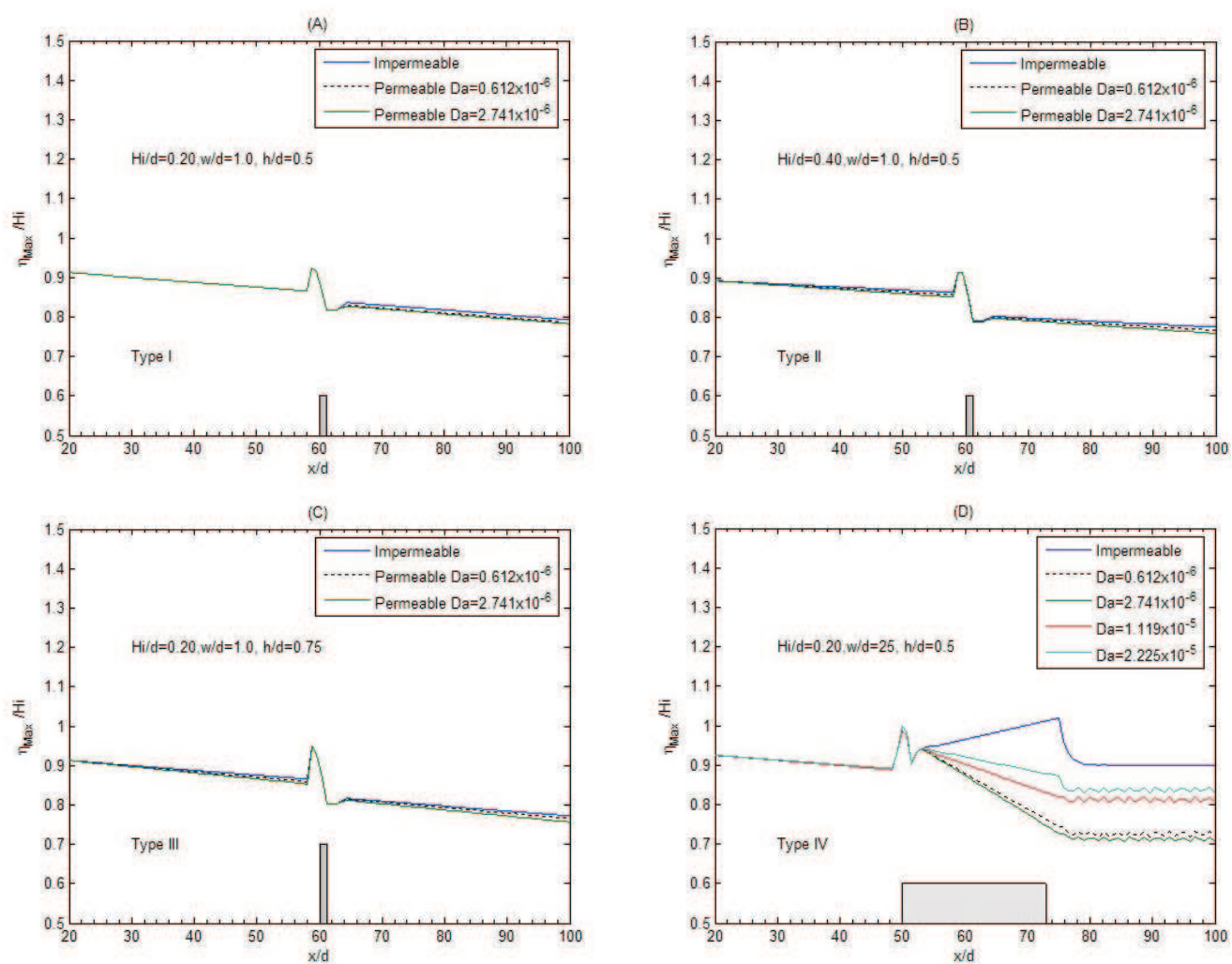

Figure 10: The maximum water surface elevation as a solitary wave passes over a submerged porous breakwater.

structure, the increase of the Darcy number causes increased energy dissipation because of the flow friction within the porous structure, resulting in reducing the transmitted wave height. On the other hand, the increase in $D a$ will allow more energy penetration through the structure, thus increasing the transmitted wave height. The numerical results illustrate that for a wide porous breakwater, given a small Darcy number, an increase in $D a$ reduces the transmission coefficient; otherwise the transmission coefficient increases with $D a$.

For the case displayed in Figure 10(D), the optimum Da is approximately $2.741 \times 10^{-6}$. The results presented in Figure 10 indicate that the wave transformation is affected primarily by the breakwater width and the structure permeability. Figure 11 shows the variation in the reflection coefficient with respect to the breakwater width. The reflection coefficient is defined as the reflected wave height divided by the incident wave height $\frac{\eta_{r}}{H_{i}}$. Hence, an optimum $D a$ value seems to exist, at which the transmitted wave height is minimized. 


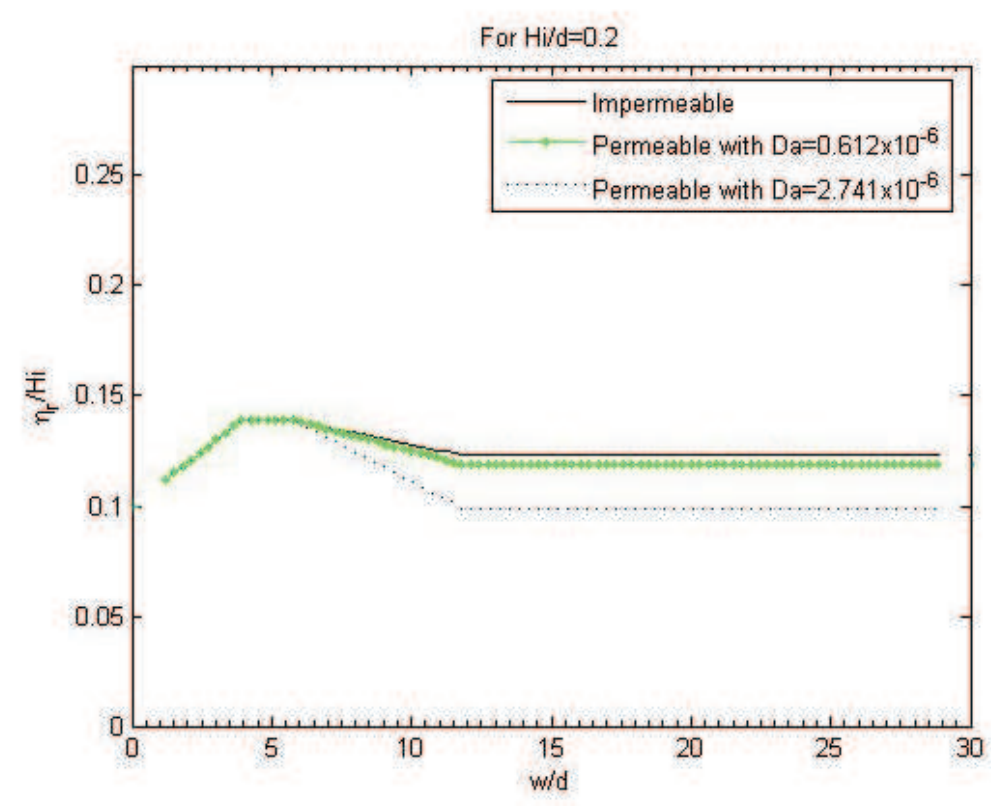

Figure 11: The variation in the reflection coefficient with respect to the breakwater width as a solitary wave propagates over the breakwater

Note from Figure 10 that when $\frac{w}{d}$ is less than or equal to 5 , the structure permeability has no apparent effect on the reflection coefficient. When $\frac{w}{d}$ is greater than 5 , the reflection coefficient decreases with increasing permeability. When $\frac{w}{d}$ is greater than or equal to 12 , the reflection coefficient remains constant, even if the breakwater width increases. The reflection coefficient increases apparently with the breakwater height with its value from 0.11 for $\frac{h}{d}=0.50$ to 0.26 for $\frac{h}{d}=0.70$ when $\frac{w}{d}=1$. The reflection coefficient decreases slightly with the increase in the incident wave height. Figure 12 shows the variations in the transmission coefficient with respect to the breakwater width. The transmission coefficient is defined as the ratio of the transmitted wave height at the trailing edge to the incident wave height. We noted that the transmission coefficient increases with the breakwater width for the impermeable case due to the nonlinear effect, while it decreases in the permeable cases. The transmission coefficient decreases as Darcy number $D a$ increases from $0.612 \times 10^{-6}$ to $2.741 \times 10^{-6}$. However, the results in Figure 10(D) indicate that the further increase of the structure permeability will result in the increase of the transmission coefficient.

\section{Conclusion}

In this paper, the new model unsteady two-dimensional incompressible Navier-StokesBrinkman system were solved numerically to simulate the propagation of non-linear wave 


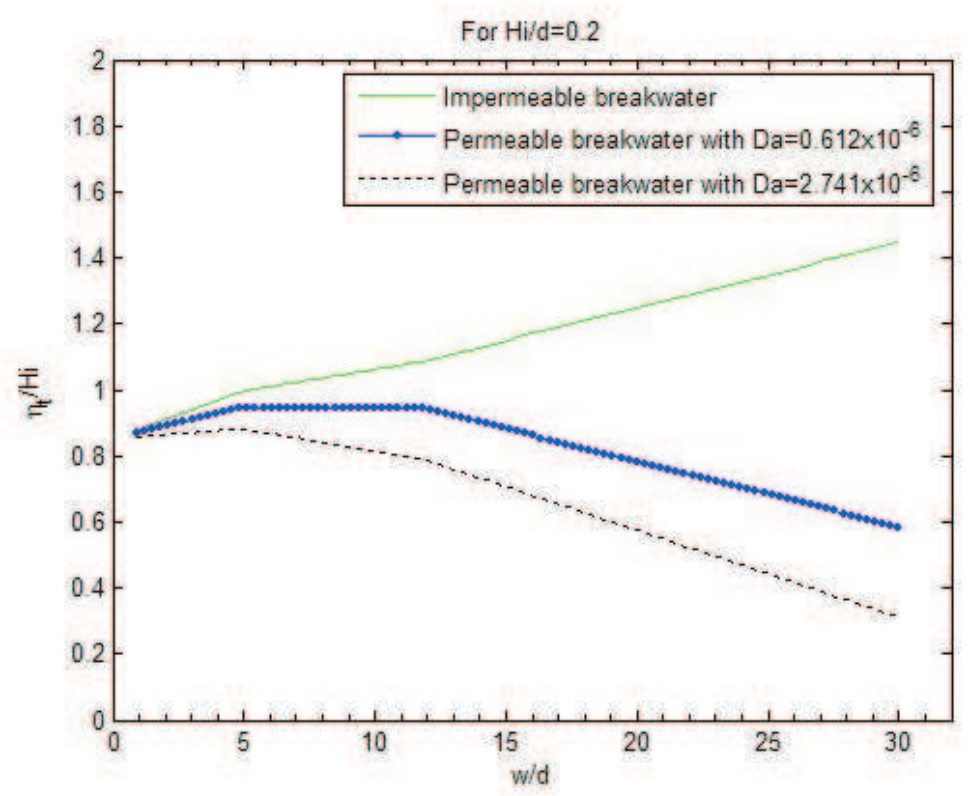

Figure 12: The variation in the transmission coefficient with respect to the breakwater width as a solitary wave propagates over the breakwater

over submerged porous structure. The staggered grid finite volume method is used to approximate the system. A volume of fluid method is used to simulate free surface flows. We validate the proposed numerical model with the theoretical and experimental solution. At first, the numerical experiments are performed with and without porous breakwater. Close agreements between numerical and experimental results show the robustness of the new model. Effect of different parameters (The incident wave height, structure permeability, length and width of the breakwater) on the wave height attenuation were studied and discussed. The numerical results also illustrate that for the porous breakwater, an optimum porosity value seems to exist at which the transmitted wave height is minimized. It has been observed that the transmission coefficient decreases as the permeability increases. However, the results indicate that further increase of the structure permeability beyond the optimal value increase the transmission coefficient. It is found that, if the breakwater is considered as a porous medium, the waves start decaying in front of a distance of the porous breakwater, then decreasing rapidly over the breakwater, and decaying continuously at the back of the breakwater, due to the permeability and the resistance of the porous medium. How ever, for the case of the impermeable structure, the waves only start decaying as the waves passing over the breakwater and it is found that the recovery waves will appear at a distance behind the breakwater. 


\section{References}

[1] M. H. Abdul Khader and S. P. Rai, A study of submerged breakwaters, Journal of Hydraulic Research, 18(1980), 113-121.

[2] A. Chiranjeevi Rambabu and J. S. Mani, Numerical prediction of performance of submerged breakwaters, Journal of Ocean Engineering, 32(2005), 1235-1246.

[3] A. T. Chwang and W. Li, A piston-type porous wavemaker theory, Journal of Engineering Mathematics, 17 (1983), 301-313.

[4] Ching-Jer Huang and Chih-Ming Dong, On interaction of a solitary wave and a submerged dike, Coastal Engineering, 43(2001), 265-286.

[5] C. W. Hirt and B. D. Nichols, Volume of Fluid (VOF) method for the dynamics of free boundaries, Journal of Computational Physics, 39(1981), 201-225.

[6] M. M. K. Darwiche, A. N. Willians and K. H. Wang, Wave interaction with semi-porous cylindrical breakwater, Journal of Waterway, Port, Coastal and Ocean Engineering, 120 (1994), 382-403.

[7] Dong-Soo Hur, Chang-Hoon Kim, Do-Sam Kim and Jong-Sung Yoon, Simulation of the nonlinear dynamic interactions between waves, a submerged breakwater and the seabed, Journal of Ocean Engineering, 35(2008), 511-522.

[8] C. J. Huang, E. C. Zhang and J. F. Lee, Numerical simulation of nonlinear viscous wavefields generated by a piston-type wavemaker, Journal of Engineering Mechanics, 124(1998), $1110-1120$.

[9] G. H. Keulegan, Gradual damping of solitary waves, J. Res. Natl. Bur. Stand, 40(1948), 487-498.

[10] C. C. Mei, The applied Dynamics of Ocean Surface Waves, Wiley, New York, 1983.

[11] J. S. Russell, Report of the committe on waves, Rep. Meet. Brit. Assoc. Adv.Sci. 7th. Liverpool, 1837, John Murray, 1838, 417-496.

[12] S. Mohan Kumar, S. Sundar and V. Vetrivel, Navier-Stokes-Brinkman model for incompressible flow over a porous Layer, International Workshop on Modeling and Simulation, Narosa, 2004, 283-293.

[13] K. H. Wang and Xugui Ren, Water waves on flexible and porous breakwaters, Journal of Engineering Mechanics, 119(1993), 1025-1047.

[14] W. J. Moyce and J. C. Martin, An experimental study of the collapse of fluid columns on a rigid horizontal plane, in a medium of lower, but comparable, density, Mathematical and physical Sciences, 224(1952), 325-334.

[15] X. Yu and A. T. Chwang, Wave-induced oscillation in harbor with porous breakwaters, Journal of Waterway Port Coastal Ocean Engineering, 120 (1994), 125-144.

Department of Mathematics, Indian Institute of Technology Madras, Chennai600 036, India.

E-mail: senalemi2007@gmail.com

Department of Mathematics, Indian Indian Institute of Technology Madras, Chennai600 036 , India. 